\title{
DERIVATIONS WITH NILPOTENT VALUES ON LIE IDEALS
}

\author{
CHARLES LANSKI
}

(Communicated by Donald S. Passman)

\begin{abstract}
Let $R$ be a ring containing no nonzero nil right ideal and let $U$ be a Lie ideal of $R$. If $d$ is a derivation of $R$ so that $d(u)$ is a nilpotent element for each $u \in U$, then $d=0$ when $R$ is a prime ring and $U$ is not commutative. The main result shows that in general, $d(I)=0$ for $I$ the ideal $R$ generated by $[U, U]$ and that $R$ is the subdirect sum of two images so that $d$ induces the zero derivation on one, and the image of $U$ in the other is commutative.
\end{abstract}

This paper continues a line of investigation in the literature concerning derivations having nilpotent values. The first such result, which inspired all those which followed, is due to I. N. Herstein [6] who proved that if $R$ is a prime ring and $d$ is an inner derivation of $R$ satisfying $d(x)^{n}=0$ for all $x \in R$ and $n$ a fixed integer, then $d=0$. This result was extended to arbitrary derivations in semiprime rings in [3]. In [2], the authors considered derivations satisfying $d(x)^{n(x)}=0$ for all $x \in I$, an ideal of $R$, and proved that $d(I)=0$ when $R$ has no nil right ideal. Using the general approach in [2], Carini and Giambruno [1] study the situation when $d(x)^{n(x)}=0$ for all $x \in U$, a Lie ideal of $R$. They prove first that $d(U)=0$ when $R$ is a prime ring, char $R \neq 2$, and $R$ contains no nil right ideal, and then obtain the same conclusion when $n(x)=n$ is fixed and $R$ is a 2 -torsion free semiprime ring. Our purpose here is to remove both the bound on the indices of nilpotence and the characteristic assumptions on $R$. We rely on the ideas in [1] to handle the semisimple case, and the methods in [2] to complete the proof in general.

Throughout this paper let $R$ denote a ring containing no nonzero nil right ideal, $U$ a Lie ideal of $R, Z$ the center of $R$, and $d$ a derivation of $R$. For any nonempty subset $A$ of $R$, call $d$ nil on $A$ if $d(a)^{n(a)}=0$ for each $a \in A$. It is clear that to conclude that $d=0$, or that $d(U)=0$, when $d$ is nil on $U$, one must assume that $R$ does not contain a nil ideal $I$, since otherwise for any $y \in I,[r, y]=d_{y}(r) \in I$ is nilpotent and so $d_{y}$ is nil on $R$. The reason why we must assume that $R$ contains no nil right ideal is given in [2]: If $d$ is nil

Received by the editors September 23, 1988 and, in revised form, February 23, 1989.

1980 Mathematics Subject Classification (1985 Revision). Primary 16A72; Secondary 16A68, $16 \mathrm{~A} 12$. 
on an ideal $I$, then the assumption of no nil ideals in $R$ will force $d(I)=0$ if and only if the Koethe conjecture is true.

Some well-known facts about Lie ideals are necessary in our arguments, so for convenience of reference we record a few of them as remarks. Recall that a Lie ideal is an additive subgroup $U$ of $R$ so that $[u, r]=u r-r u \in U$ for all $r \in R$.

Remark 1. If $R$ is a prime ring and $U$ is a commutative Lie ideal, that is $[U, U]=0$, then either $U \subset Z$, or $R$ satisfies the standard polynomial identity $S_{4}$ and $\operatorname{char} R=2$ [7; Theorem 4, p. 118].

Remark 2. If $U$ is a noncommutative Lie ideal of $R$ and if $I=([U, U])$, the ideal of $R$ generated by $[U, U]=\{[x, y] \mid x, y \in U\}$, then $I \subset U+U^{2}$ and $[I, R] \subset U[4$; proof of Lemma 1.3, p. 4].

Remark 3. If the elements of $U$ are nilpotent then $U=0$. To see this, let $u \in$ $U-\{0\}$ with $u^{k}=0$ and $k$ minimal. Set $v=u a u^{k-1}=\left[u, a u^{k-1}\right] \in U$ for any $a \in R$, and note that $v^{2}=0$. Now for $r \in R, 0=[v, r]^{n(r)} v r=(v r)^{n(r)+1}$, so $v R$ is a nil right ideal of $R$ forcing $v=0$. Thus $U=0$ as claimed.

Remark 4. If $R$ is any ring, $d$ a derivation of $R, U$ a Lie ideal of $R, T$ a right ideal of $R$, and $J$ an ideal of $R$, then $U+d(U)$ is a Lie ideal of $R, T+d(T)$ is a right ideal of $R$, and $J+d(J)$ is an ideal of $R$.

We also require a couple of easy preliminary lemmas. The first of these is essentially [2; Lemma 1, p. 522] or [1; Remark 1, p. 501] adapted to our needs.

Lemma 1. If $x, y \in R$ with $x y=0, I=([U, U])$, and $d$ is nil on $U$, then $x d(y) I$ is a nil right ideal of $R$. In particular, when $U$ is noncommutative then $x d(y)=d(x) y=0$ if either $R$ is a prime ring or if $x \in I$.

Proof. For all $t \in I, y t x=[y t, x] \in[I, R] \subset U$. Thus for some $k=k(t)$, $x d(y t x)^{k} d(y) t=0$, and using $d(y t x)=d(y) t x+y d(t x)$ yields $(x d(y) t)^{k+1}=$ 0 , proving that $x d(y) I$ is a nil right ideal. Now if $U$ is not commutative then $I \neq 0$ and $x d(y)=0$ when $R$ is a prime ring. When $x \in I$, then $x d(y) \in I \cap \operatorname{ann}(I)=0$. In either case $d(x y)=0$ gives $d(x) y=0$ as well.

Our second lemma will be important in transfering what we can prove for prime rings to semiprime rings.

Lemma 2. Let $S$ be any ring, $V$ a Lie ideal of $S$, and $P$ a prime ideal of $S$ so that $S / P$ contains no nonzero nil right ideal. If $d$ is derivation of $S$ which is nil on $V$, then either $d(P) \subset P$ or $d(V) \subset P$.

Proof. Suppose that $d(P) \not \subset P$ so that $d(P)+P$ is a nonzero ideal of $S / P$ by Remark 4. Now $[V, P] \subset V$, so $d[V, P]+P=[V, d(P)+P]+P$ is a nil Lie ideal in $S / P$ and it follows from Remark 3 that $[V, d(P)+P] \subset P$. Since $d(P)+P$ is an ideal in the prime ring $S / P$, its centralizer is central, and $V+P \subset Z(S / P)$ results. By using $[V, S] \subset V$, one sees that $d[V, S]+$ $P=[d(V)+V, S]+P$ is a nil Lie ideal in $S / P$, so again Remark 3 forces 
$d(V)+P \subset Z(S / P)$. But $d(V)$ consists of nilpotent elements, so we must have $d(V) \subset P$, proving the lemma.

The first theorem in [1] shows that when $R$ is a primitive $\operatorname{ring}$, char $R \neq 2$, and $d$ is nil on $U$, then $d=0$. The proof of this result, as well as of the two lemmas preceding it in [1], hold exactly as given without the assumption on char $R$, if $U$ is noncommutative. These proofs only require $[I, R] \subset U$ for a nonzero ideal $I$ of $R$, which holds by Remark 2. This observation, together with our Lemma 2 above, leads to our first theorem which is essentially [1; Theorem 2, p. 500]. Our proof is somewhat easier than that in [1], and more importantly, avoids the torsion assumption.

Theorem 1. Let $R$ be a semisimple ring, suppose that $d$ is nil on $U$, and set $I=([U, U])$. Then $d(I)=0$, and $d(U)=0$ if $R$ is 2-torsion free.

Proof. Let $P$ be a primitive ideal of $R$. Since $R$ is a semisimple ring, the intersection of its primitive ideals is zero. Furthermore, it is easy to see that if $R$ is 2 -torsion free then those primitive ideals $Q$ of $R$ satisfying char $R / Q \neq 2$ have intersection equal to zero, so in this case we may assume that char $R / P \neq$ 2. Clearly, Lemma 2 applies and we conclude that either $d(U) \subset P$ or $d(P) \subset$ $P$. The latter possibility means that $d$ induces the derivation $D$ on $R / P$ with $D$ nil on $U+P$. If $U+P$ is noncommutative in $R / P$ then $D=0$ by [1; Theorem 1, p. 499] and our comment above, so in particular $d(U) \subset P$. Should $U+P$ be commutative and char $R / P \neq 2$, then $U+P \subset Z(R / P)$ by Remark 1. Thus, $d(U)+P$ are nilpotent elements in $Z(R / P)$, forcing $d(U) \subset P$. This proves that $d(U)=0$ when $R$ is 2-torsion free. If $U+P$ is commutative and char $R / P=2$, then $I=([U, U]) \subset P$, so $d(I) \subset d(P) \subset P$. In those cases when $d(U) \subset P$, using Remark 2, $d(I) \subset d\left(U+U^{2}\right) \subset d(U)+d\left(U^{2}\right) \subset P$. Therefore, $d(I) \subset P$ always holds, proving that $d(I)=0$, as required.

Using Theorem 1 in place of [1; Theorem 2, p. 500] one can get a characteristic free version of [1; Theorem 3, p. 502], which we state for convenience.

Theorem 2. If $R$ is a prime ring containing no nil right ideal, $U$ is a noncommutative Lie ideal of $R$, and $d$ is nil on $U$, then $d=0$.

Proof. When $R$ is semisimple, Theorem 1 shows that $d(I)=0$ for $I=$ $([U, U])$. Since $I \neq 0$ and $R$ is a prime ring it follows easily that $d=0$. On the other hand, if the Jacobson radical of $R$ is not zero, then the proof of [1; Theorem 3, p. 502] holds exactly as given to show that $d(U)=0$. Hence $d(I) \subset d\left(U+U^{2}\right)=0$, by Remark 2 , and as above $d=0$ follows.

Before we state our main result, whose proof is rather long and complex, let us examine a little more closely when $d$ can be nil on $U$ in a prime ring without nil right ideals. By Theorem 2, unless $d=0, U$ must be commutative. If $U$ is central then $d(U)=0$ and the condition that $d$ is nil on $U$ is a trivial one. Finally, if $U$ is commutative but not central then by Remark 1 char $R=2$ and $R$ satisfies $S_{4}$. Should $R$ be a domain then $d(U)=0$ and again, the 
assumption that $d$ is nil on $U$ is trivial. Assuming that $R$ has zero divisors, it is an order in $M_{2}(F)$, for $F$ a field with char $F=2$ [7; Theorem 4, p. 118]. For simplicity, and to see how examples may arise, let us assume that $R=M_{2}(F)$. The nature of $U$ forces $u^{2} \in F$ for all $u \in U$, and this in turn forces $U \subset[R, R]$, which is 3-dimensional over $F$. It is not hard to show that $U$ must have the form $U=F+F\left(\begin{array}{ll}0 & a \\ b & 0\end{array}\right)$. If $d$ is nil on $U$, then it is nil on $F$, so $d(F)=0$ and $d$ must be an inner derivation. To obtain a specific example, one need only take $d$ to be defined by $A \in R$ which satisfies the condition that $\left[A,\left(\begin{array}{ll}0 & a \\ b & 0\end{array}\right)\right]$ is nilpotent. For example, if $U=F+F\left(\begin{array}{ll}0 & a \\ a & 0\end{array}\right)$, then take $A=\left(\begin{array}{ll}1 & 1 \\ 0 & 0\end{array}\right)$, or if $U=F+F\left(\begin{array}{ll}0 & a \\ 0 & 0\end{array}\right)$, then $A$ can be $\left(\begin{array}{ll}1 & 0 \\ 0 & 0\end{array}\right)$.

The preceding remarks show that the assumption that $d$ is nil on $U$ can arise in prime rings in essentially three ways: when $d=0$; when $U \subset Z$ and $d(U)=0$; and when $R \subset M_{2}(F)$ as described above. Certainly one can obtain examples of semiprime rings by taking direct sums of such prime rings. Consequently, it is necessary in the conclusion of our main theorem to allow $d(U)=0$ without $d=0$, or $d(I)=0$ without $d(U)=0$, unless one assumes that $R$ is 2 -torsion free.

Theorem 3. Let $R$ be a ring containing no nonzero nil right ideal, $U$ a Lie ideal of $R, I=([U, U])$, and $d$ a derivation of $R$ which is nil on $U$. Then $d(I)=0$, and if $R$ is 2-torsion free then $d(U)=0$. Furthermore, $R$ is $a$ subdirect sum of images $R_{1}$ and $R_{2}$ containing no nonzero nil right ideals, $d$ induces derivations $d_{i}$ on $R_{i}, d_{1}=0$, and the image of $U$ in $R_{2}$ is commutative (central if $R$ is 2-torsion free).

Proof. We show first that $I d(I)=0$, which is by far the longest and most difficult part of the proof. Since $R$ is a semiprime ring, it suffices to prove that $I d(I) \subset P$ for each prime ideal $P$ of $R$, and clearly, we may assume that $I \neq 0$. If $R$ is a semisimple ring, then $d(I)=0$ by Theorem 1 , and certainly $I d(I)=0$. Thus $J$, the radical of $R$ is not zero.

Let $P$ be any prime ideal of $R$ and assume that $R / P$ contains no nonzero nil right ideal. Then from Lemma 2 we may conclude that either $d(U) \subset P$ or $d(P) \subset P$, and the first possibility yields $d(I) \subset d\left(U+U^{2}\right) \subset P$, using Remark 2. When $d(P) \subset P, d$ induces the derivation $D$ on $R / P$ and $D$ is nil on $U+P$, so $D=0$ if $U+P$ is noncommutative by Theorem 2 . In this case $d(I) \subset P$ clearly holds. Should $U+P$ be commutative, then $I=([U, U]) \subset P$, and again $I d(I) \subset P$. Note that any primitive ideal of $R$ satisfies the condition that $R / P$ has no nil right ideal, so our argument shows that $I d(I) \subset J$.

It remains to show that $I d(I) \subset P$ when $R / P$ contains a nonzero nil right ideal, and to do so we proceed as in [2; Theorem 9, p. 527]. Set $R^{\prime}=R / P$, $r^{\prime}=r+P$ for $r \in R$, and let $K^{\prime}$ denote a nonzero nil right ideal in $R^{\prime}$. If $J^{\prime}=0$, then $I d(I) \subset J \subset P$, and we have established our claim. Therefore, we may assume that $J^{\prime}$ is a nonzero ideal of the prime ring $R^{\prime}$, and so, $J^{\prime}$ is itself a prime ring and contains the nonzero nil right ideal $K^{\prime} J^{\prime}$. Next, let $M^{\prime}$ be 
the set theoretic union of all nil right ideals in $J^{\prime}$, and set $T=\{t \in J \mid$ atb $=0$ whenever $a, b \in I$ and $a b=0\}$. Observe that $T$ is a subring of $J$ and is invariant with respect to $J$ in the sense that for $x \in J$ with quasi-inverse $y$, and $t \in T,(1+x) t(1+x)^{-1}=t+x t+t y+x t y \in T$. In particular, if $m^{\prime} \in M^{\prime}$ with $m^{\prime}=x+P$ for $x \in J$, then $T^{\prime}$ is a subring of $J^{\prime}$ which is invariant with respect to the elements of $M^{\prime}$. This is precisely the situation in which we may apply a theorem of Herstein [5; Theorem, p. 206] to conclude that either $A^{\prime} \subset T^{\prime}$ for $A^{\prime}$ a nonzero ideal of $J^{\prime}$, or $T^{\prime} \subset Z\left(J^{\prime}\right)$.

Assume first that $A^{\prime} \subset T^{\prime}$, so that $H^{\prime}=J^{\prime} A^{\prime} J^{\prime} \subset T^{\prime}$ for $H^{\prime}$ a nonzero ideal of $R^{\prime}$. Now let $x \in I$ be nilpotent of index $n$. From the definition of $T$, one gets $0=x^{n-1} T x=x^{n-2} T x T x=\cdots=(x T)^{n}$. Hence $\left(x^{\prime} T^{\prime}\right)^{n}=0$, which implies that $x^{\prime} H^{\prime}$ is a nilpotent ideal of $R^{\prime}$. Consequently, $x^{\prime}=0$, and so, all nilpotent elements of $I$ are in $P$. But $d[I, I] \subset I \cap d(U)$ are nilpotent elements, so $d[I, I] \subset P$. Take $x, y \in I$ and $v \in[I, I]$ and compute $d[x v, y]=(d[x, y]) v+[x, y] d(v)+d(x)[v, y]+x d[v, y]$. Using $d[I, I] \subset P$, $x v, y, x \in I$ and $v \in[I, I]$, results in $d(I)[[I, I], I] \subset P$. In particular, $d\left(I^{2}\right)[[I, I], I] \subset P$ so it follows that $d(I) I[[I, I], I] \subset P$. If $d(I) I \not \subset P$, then $[[I, I], I] \subset P$, which implies that $\left[I^{\prime}, I^{\prime}\right] \subset Z\left(R^{\prime}\right)$ because $I^{\prime}$ is a nonzero ideal in $R^{\prime}$. For $x, y \in I^{\prime}$, observe that $0=[[x, x y], y]=[x[x, y], y]=$ $[x, y]^{2}$, which shows that $I^{\prime}$ is commutative, since $Z\left(R^{\prime}\right)$ is a domain. Now for $v, w \in R^{\prime}$, we have first that $0=[x, y w]=y[x, w]$, so $I^{\prime} \neq 0$ forces $I^{\prime} \subset Z\left(R^{\prime}\right)$, and then, $0=[x w, v]=x[w, v]$. This yields the fact that $R^{\prime}$ is a commutative prime ring, contradicting $K^{\prime} \neq 0$. Therefore, we must have $d(I) \subset P$, which forces $I d(I) \subset P$, using the primeness of $R^{\prime}$ again. This finishes the case of $A^{\prime} \subset T^{\prime}$.

Now we must show that $I d(I) \subset P$ when $T^{\prime} \subset Z\left(J^{\prime}\right)$. Of course $T^{\prime} \subset Z\left(R^{\prime}\right)$ as well, since $R^{\prime}$ is a prime ring. To use this assumption, we must find some elements in $T^{\prime}$, and we do so next. We are assuming that $I \neq 0$, so by Lemma 1 , if $x, y \in I$ and $x y=0$, then $d(x) y=0$. Let $a, b \in I$ with $a b=0$, and suppose that $x \in J$ so that $d(x) \in J$ also. Now $a(1+x)(1+x)^{-1} b=0$ and both $a(1+x)$ and $(1+x)^{-1} b$ are in $I$, resulting first in $d(a(1+x))(1+x)^{-1} b=$ 0 , and then $a d(x)(1+x)^{-1} b=0$. The definition of $T$ and our assumption on $x$ show that $d(x)(1+x)^{-1} \in T$, so if $d(x)^{\prime}$ is a zero divisor in $R^{\prime}$, then $d(x)^{\prime}=0$ because $T^{\prime} \subset Z\left(R^{\prime}\right)$. In particular, if $x \in U \cap J^{2}$, then $d(x) \in J \cap d(U)$ is nilpotent, and so we have shown that $d\left(U \cap J^{2}\right) \subset P$. Now for $x \in I$ and $y \in I J^{2},[x, y]$ and $[x y, y]=[x, y] y$ are in $U \cap J^{2}$. Thus, $d([x, y] y)=d([x, y]) y+[x, y] d(y) \in P$, which yields $[I, y] d(y) \subset P$ for any $y \in I J^{2}$. Using the fact that $[, y]$ is a derivation results in $[I, y] I d(y) \subset P$. If $I \not \subset P$, then either $d(y) \in P$ or $[I, y] \subset P$ for each $y \in I J^{2}$, and so $I J^{2}$ is the union of two additive subgroups, forcing it to be one or the other. That is, either $d\left(I J^{2}\right) \subset P$ or $\left[I, I J^{2}\right] \subset P$. 
Consider the case that $\left[I, I J^{2}\right] \subset P$, but $I \not \subset P$. Since we know that $J \not \subset P$, and so $I J^{2} \not \subset P$, it follows that $W=I J^{2}+P$ is a nonzero ideal in $R^{\prime}$ satisfying $[W, W]=0$. As in the similar situation above arising in the case $A^{\prime} \subset T^{\prime}$, it follows that $R^{\prime}$ is commutative, contradicting $K^{\prime} \neq 0$. Consequently, $\left[I, I J^{2}\right] \subset P$ requires $I \subset P$, and so leads to $I d(I) \subset P$. Finally, if $d\left(I J^{2}\right) \subset P$, then also $d\left(I J^{2} R\right) \subset P$, resulting in $I J^{2} d(R) \subset P$. In this case, either $I \subset P$ or $d(R) \subset P$ so that $I d(I) \subset P$ must hold. This completes the proof of the fact that $I d(I)=0$ as claimed.

Using $I d(I R)=0$ leads to $I^{2} d(R)=0$, and so $d(R) \subset \operatorname{ann}\left(I^{2}\right)=\operatorname{ann}(I)$, since $R$ is a semiprime ring. Thus for $x \in I$ and $y \in \operatorname{ann}(I), d(x) y=0$ by Lemma 1 , and it follows that $d(I) \subset \operatorname{ann}(\operatorname{ann}(I)) \cap d(R) \subset \operatorname{ann}(\operatorname{ann}(I)) \cap$ $\operatorname{ann}(I)=0$, proving the first statement of the theorem. Now, the definition of $I$ yields both $d[U, U]=0$ and $d([U, U] R)=0$ and combining these shows that $[U, U] \subset \operatorname{ann}(B)$, where $B=(d(R))$ is the ideal of $R$ generated by $d(R)$. It is clear that $B$ and its annihilator are invariant under $d$, so $R_{1}=R / \operatorname{ann}(\operatorname{ann}(B))$ and $R_{2}=R / \operatorname{ann}(B)$ have derivations $d_{i}$ induced by $d$. Certainly, $d_{1}=0$, and since $[U, U] \subset \operatorname{ann}(B)$, the image of $U$ in $R_{2}$ is commutative. It is straightforward to check that neither $R_{1}$ nor $R_{2}$ contains a nonzero nil right ideal; in fact, $R / \operatorname{ann}(A)$ has this property for any ideal $A$ of $R$. Finally, when $R$ is 2-torsion free, it is easy to check that $R_{2}$ is also, and given the properties of $R_{2}$, that there is a collection $W$ of prime ideals of $R$ so that $\operatorname{ann}(B)=$ $\bigcap\{Q \in W\},[U, U] \subset Q$ for each $Q \in W$, and $\operatorname{char}(R / Q) \neq 2$. By Remark 1 , $U+Q \subset Z(R / Q)$, and so $d[U, R]+Q=[d(U)+U, R]+Q$ is a nil Lie ideal of $R / Q$ using Remark 4. But then, Remark 3 forces $d(U)+Q \subset Z(R / Q)$ and the fact that these elements are nilpotent shows that $d(U) \subset Q$. Therefore, when $R$ is 2-torsion free, the image of $d(U)$ in $R_{2}$ is zero, so $d(U)=0$, completing the proof of the theorem.

As in both [1] and [2], it is possible to replace the assumption of no nil right ideal in $R$ with other conditions. One could assume that $R$ has no nil ideal together with some property which precludes the possibility of a nil right ideal: for example, if $R$ is an algebra over an uncountable field or if nil subrings are locally nilpotent. Finally, if $d$ is nil on $U$ of bounded index, which means that $d(u)^{n}=0$ for $n$ independent of $u \in U$, then it suffices to assume that $R$ is semiprime as in [1] or [3]. To see why this is so, note first that Levitzki's theorem [4; Lemma 1.1, p. 1] shows that in a semiprime ring, any nil right ideal of bounded index is zero. Using this, Remark 3 holds for a nil Lie ideal of bounded index in a semiprime ring, and Lemma 1 holds when $d$ is nil of bounded index in $U$. A quick examination of our proofs, as well as those in [1] and [2] on which they depend, shows that the only use made of the assumption that $R$ has no nil right ideal is in applying either Remark 3 or Lemma 1. Thus our main result holds in this case, and we finish with its statement. 
Theorem 4. Let $R$ be a semiprime ring, $U$ a Lie ideal of $R, I=([U, U])$, and $d$ a derivation of $R$ which is nil of bounded index on $U$. Then $d(I)=0$, and if $R$ is 2-torsion free then $d(U)=0$. Furthermore, $R$ is a subdirect sum of semiprime images $R_{1}$ and $R_{2}, d$ induces derivations $d_{i}$ on $R_{i}, d_{1}=0$, and the image of $U$ in $R_{2}$ is commutative (central if $R$ is 2-torsion free).

\section{REFERENCES}

1. L. Carini and A. Giambruno, Lie ideals and nil derivations, Bollettino U.M.I., 6 (1985), 497503.

2. B. Felzenszwalb and C. Lanski, On the centralizers of ideals and nil derivations, J. Algebra 83 (1983), 520-530.

3. A. Giambruno and I. N. Herstein, Derivations with nilpotent values, Rend. Circ. Mat. Palermo 30 (1981), 199-206.

4. I. N. Herstein, Topics in ring theory, University of Chicago Press, Chicago, 1969.

5. __, Invariant subrings of a certain kind, Israel J. Math. 26 (1977), 205-208.

6. __ Center-like elements in prime rings, J. Algebra 60 (1979), 567-574.

7. C. Lanski and S. Montgomery, Lie structure of prime rings of characteristic 2, Pacific J. Math. 42 (1972), 117-136.

Department of Mathematics, University of Southern California, Los Angeles, CALIFORNIA 90089 